\title{
Helium abundances in planetary nebulae: Nucleosynthesis and chemical evolution
}

\author{
W. J. Maciel, R. D. D. Costa and T. E. P. Idiart \\ University of São Paulo, Astronomy Department, \\ Rua do Matão 1226, Cidade Universitária, São Paulo SP, CEP 05509-0900, Brazil \\ email: maciel@astro.iag.usp.br, roberto@astro.iag.usp.br, thais@astro.iag.usp.br

\begin{abstract}
We have obtained a large sample of PN with accurately determined helium abundances, as well as abundances of several heavy elements. The nebulae are located in the solar neighbourhood, in the galactic bulge, disk and anticentre, and in the Magellanic Clouds. The abundances are analyzed both in terms of the nucleosynthesis of intermediate mass stars and the chemical evolution of the host galaxies. In particular, correlations between the $\mathrm{He} / \mathrm{H}$ ratio and the abundances of $\mathrm{N}$ and $\mathrm{O}$ are used as constraints of the nucleosynthetic processes occurring in the progenitor stars.
\end{abstract}

Keywords. ISM: planetary nebulae: general; Galaxy: abundances

\section{Introduction}

Helium abundances can be accurately measured in photoionized nebulae, comprising planetary nebulae $(\mathrm{PN})$ and HII regions. The abundances measured in PN include the original helium content previous to the formation of the progenitor stars and the contamination during the nuclear processes in these objects. As a consequence, PN can be used to study the nucleosynthetic processes in intermediate mass stars and the chemical evolution of the host systems. In this work, we analyze the helium abundances of a large sample of PN in different systems, namely, the disk and bulge of the Milky Way (MW), and both the Small (SMC) and Large (LMC) Magellanic Clouds.

\section{Average abundances and abundance distributions}

We have analyzed several samples of $\mathrm{PN}$ with elemental abundances obtained in a homogeneous way. We have considered our own data, the IAG sample (see Maciel et al. 2009 and references therein, and Cavichia et al., in preparation), plus some recent data from the literature (Stasińska et al. 1998, SRM, Leisy \& Dennefeld 2006, LD). Maciel et al. (2009) made a detailed comparison of these results concerning the heavy elements, and concluded that the merged sample maintains the homogeneity of the individual samples, in view of the similar methods employed. Average helium abundances are in the range $0.093 \leqslant \mathrm{He} / \mathrm{H} \leqslant 0.119$. These averages are similar in all systems, within the uncertainties, but the SMC abundances are slightly lower than either the LMC and MW, being similar to the HII region value, as in the Orion nebula or 30 Dor. The MW disk seems slightly richer in He than the bulge, which may be due to a lack of intermediate mass stars near the upper mass limit in the latter. Concerning the $\mathrm{He} / \mathrm{H}$ distributions, there is a general agreement among the different samples. The peaks of the distributions are in the range $\mathrm{He} / \mathrm{H}=0.080$ to $0.100(\mathrm{SMC})$ and $\mathrm{He} / \mathrm{H}=0.080$ to 0.120 (LMC), while the MW data show a peak between $\mathrm{He} / \mathrm{H}=0.120$ and 0.140 for the disk and $\mathrm{He} / \mathrm{H}=$ 0.080 to 0.120 for the bulge. 

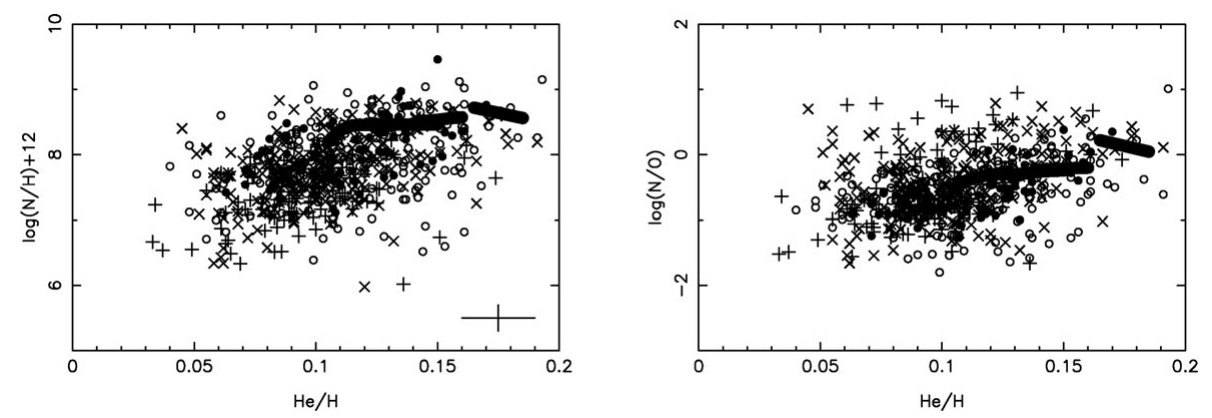

Figure 1. He and $\mathrm{N}$ abundances in PN in the Milky Way disk (dots), Bulge (empty circles), SMC (crosses) and LMC (x signs). Thick lines are models for TP-AGB stars with masses in the range 1.1 to $5 M_{\odot}$ by Marigo et al. (2003).

\section{Abundance correlations: Observations and theory}

Figure 1 shows the N/H vs $\mathrm{He} / \mathrm{H}$ and N/O vs $\mathrm{He} / \mathrm{H}$ plots for the merged sample. A few objects with $\mathrm{He} / \mathrm{H}<0.03$ are not included, as some contribution from neutral helium is probably unaccounted for. It is apparent that all systems have a similar behaviour, with similar results for the $\mathrm{N} / \mathrm{O}$ ratio, although the metallicity range may be different in these systems. For example, the MW shows a larger range in nitrogen abundances, as expected, since our galaxy is more metal rich than the Magellanic Clouds. However, the evolution of the abundances is consistent with a similar trend for all systems, since the slopes are similar. In Fig. 1 we have included the predictions of theoretical models by Marigo et al. (2003), as shown by the thick lines. These are synthetic evolutionary models for the thermally-pulsing Asymptotic Giant Branch stars (TP-AGB) with masses in the range 1.1 to $5 M_{\odot}$. In these objects, up to three dredge-up episodes occur, apart from hot-bottom burning (HBB) for the most massive objects. All of these processes affect the $\mathrm{He} / \mathrm{H}$ ratio, and in fact most objects present some enhancement compared to the solar values. According to Marigo et al. (2003), progenitors having 0.9 to $4 M_{\odot}$ and solar composition can explain the "normal" abundances, $\mathrm{He} / \mathrm{H} \leqslant 0.15$, while for those objects with higher enhancements $(\mathrm{He} / \mathrm{H}>0.15)$ larger masses are needed, in the range 4 to $5 M_{\odot}$, plus an efficient HBB. The latter are represented in Fig. 1 by the slanted black thick lines at the upper right corner of the figures. It can be seen that the agreement is very good, especially for the $\mathrm{N} / \mathrm{O}$ ratio. For the $\mathrm{N} / \mathrm{H}$ ratio the plot shows a better agreement for the Milky Way, as expected, since this galaxy is somewhat more metal rich than the Magellanic Clouds, so that we would expect the theoretical predictions to be located in the upper part of the plot, as is indeed the case. We found a continuous transition between the "normal" and He-richer nebulae, which is probably due to the fact that we have a much larger sample compared to Marigo et al. (2003). These results suggest that the nucleosynthetic processes occurring in these systems are similar, even though the global metallicity may be different and the chemical evolution may be affected by different star formation rates.

Acknowledgements. This work was partially supported by FAPESP and CNPq.

\section{References}

Leisy, P. \& Dennefeld, M. 2006, A\& A, 456, 451

Maciel, W. J., Costa, R. D. D., \& Idiart, T. E. P. 2009, Rev. Mexicana AyA, 45, 127

Marigo, P., Bernard-Salas, J., Pottasch, S. R., et al., 2003, A\&A, 409, 619

Stasińska, G., Richer, M. G., \& McCall, M. 1998, A\& A, 336, 667 